\title{
Clinical Characteristics and Changes of Clinical Features in Patients with Asthma- COPD Overlap in Korea according to Different Diagnostic Criteria
}

\author{
Jeong Uk Lim, M.D. ${ }^{1}$ (i) , Deog Kyeom Kim, M.D., Ph.D. ${ }^{2}$, Myung Goo Lee, M.D., Ph.D. ${ }^{3}$, Yong-Il \\ Hwang, M.D., Ph.D. ${ }^{4}$, Kyeong-Cheol Shin, M.D., Ph.D. , Kwang Ho In, M.D., Ph.D. ${ }^{6}$, Sang Yeub Lee, \\ M.D., Ph.D. ${ }^{7}$, Chin Kook Rhee, M.D., Ph.D. ${ }^{8}$, Kwang Ha Yoo, M.D., Ph.D. ${ }^{9}$ and Hyoung Kyu Yoon, \\ M.D., Ph.D. ${ }^{1}$ (i) \\ *Author affiliations appear at the end of this article.
}

Background: Asthma-chronic obstructive pulmonary disease (COPD) overlap (ACO) is a condition characterized by the overlapping clinical features of asthma and COPD. To evaluate the appropriateness of different sets of ACO definition, we compared the clinical characteristics of the previously defined diagnostic criteria and the specialist opinion in this study. Methods: Patients enrolled in the KOrea COpd Subgroup Study (KOCOSS) were evaluated. Based on the questionnaire data, the patients were categorized into the ACO and non-ACO COPD groups according to the four sets of the diagnostic criteria.

Results: In total 1,475 patients evaluated: 202 of 1,475 (13.6\%), 32 of 1,475 (2.2\%), 178 of 1,113 (16.0\%), and 305 of 1,250 (24.4\%) were categorized as ACO according to the modified Spanish Society of Pneumonology and Thoracic Surgery (SEPAR), American Thoracic Society (ATS) Roundtable, Global Initiative for Asthma (GINA)/Global Initiative for Chronic Obstructive Lung Disease (GOLD) criteria, and the specialists diagnosis, respectively. The ACO group defined according to the GINA/GOLD criteria showed significantly higher St. George's Respiratory Questionnaire and COPD Assessment Test scores than the non-ACO COPD group. When the modified SEPAR definition was applied, the ACO group showed a significantly larger decrease in the forced expiratory volume in 1 second $\left(\mathrm{FEV}_{1}, \%\right)$. The ACO group defined by the ATS Roundtable showed significantly larger decrease in the forced vital capacity values compared to the non-ACO COPD group $(-18.9 \%$ vs. $-2.2 \%$, $\mathrm{p}=0.007$ and $-412 \mathrm{~mL}$ vs. $-17 \mathrm{~mL}, \mathrm{p}=0.036)$. The ACO group diagnosed by the specialists showed a significantly larger decrease in the $\mathrm{FEV}_{1}(\%)$ compared to the non-ACO group $(-5.4 \%$ vs. $-0.2 \%$, $\mathrm{p}=0.003)$.

Conclusion: In this study, the prevalence and clinical characteristics of ACO varied depending on the diagnostic criteria applied. With the criteria which are relatively easy to use, defining ACO by the specialists diagnosis may be more practical in clinical applications.

Keywords: Asthma; Chronic Obstructive Lung Disease; Pulmonary Function

Address for correspondence: Hyoung Kyu Yoon, M.D., Ph.D.

Division of Pulmonary and Critical Care Medicine, Department of Internal Medicine, Yeouido St. Mary's Hospital, College of Medicine, The Catholic University of Korea, 10 63-ro, Yeongdeungpo-gu, Seoul 07345, Republic of Korea

Phone: 82-2-3779-2213, Fax: 82-2-784-5458, E-mail: cmcyhg@catholic.ac.kr

Received: Apr. 3, 2020, Revised: Jul. 25, 2020, Accepted: Oct. 13, 2020, Published online: Oct. 13, 2020

@(c) it is identical to the Creative Commons Attribution Non-Commercial License (http://creativecommons.org/licenses/by-nc/4.0/). 


\section{Introduction}

Asthma and chronic obstructive pulmonary disease (COPD) are the most common obstructive lung diseases, although they have different underlying pathophysiology and clinical manifestations ${ }^{1}$. Nevertheless, some patients show clinical features of both asthma and COPD ${ }^{2}$. Asthma-COPD overlap (ACO) is a condition characterized by overlapping clinical features of asthma and COPD. There is no consensus definition for ACO; several definitions have been proposed to categorize patients with features of both asthma and COPD. The most widely used diagnostic criteria are those of the American Thoracic Society (ATS) Roundtable ${ }^{3}$, the Spanish Society of Pneumonology and Thoracic Surgery (SEPAR) ${ }^{4}$, and the Global Initiative for Asthma (GINA)/Global Initiative for Chronic Obstructive Lung Disease (GOLD) ${ }^{5}$. Since there is currently no gold standard test to diagnosed asthma, the clinical opinion of an experienced clinician is essential ${ }^{6}$. Thus diagnosis of ACO by a specialist clinician is also important. However, the appropriateness of these criteria may differ according to the ethnic, cultural, linguistic and clinical characteristics of each country. Several Korean studies have evaluated the clinical characteristics of ACO patients ${ }^{7,8}$. However, further longitudinal studies are necessary, since the clinical characteristics and prognosis of ACO vary depending on the criteria used. Some studies have reported that ACO shows a more rapid decline in lung function ${ }^{9,10}$, although another study reported otherwise ${ }^{7}$. Additional clinical data on changes in symptom and pulmonary function test scores in ACO patients are necessary.

This retrospective study applied four sets of ACO diagnostic criteria to a cohort of Korean COPD patients. We compared clinical presentations and appropriateness of the different sets of criteria so as to know which criteria is most applicable in Korean ACO population.

\section{Materials and Methods}

\section{Diagnostic criteria for ACO}

The following diagnostic criteria for ACO were applied: (1) the ATS Roundtable criteria ${ }^{3}$, (2) the modified SEPAR criteria proposed by Soler-Cataluna et al. , (3) the GINA/GOLD criteria ${ }^{5}$, and (4) specialist diagnosis based on own experience and beliefs (clinician's diagnosis). The criteria are detailed in Supplementary Table S1.

\section{Data collection}

Patients enrolled in the KOrea COpd Subgroup Study (KOCOSS) were recruited from 48 tertiary referral hospitals in Korea, and were required to regularly visit the hospital so that their COPD status could be tracked via self-administered questionnaires, and to undergo pulmonary function tests at least every 6 months ${ }^{11}$. All data were checked for validity and coherence before evaluation. The inclusion criteria were as follows: aged $\geq 40$ years and post-bronchodilator forced expiratory volume in 1 second $\left(\mathrm{FEV}_{1}\right) /$ forced vital capacity (FVC) ratio $<0.7$.

This retrospective cohort study was initiated by the Asthma Study Group of the Korean Academy of Tuberculosis and Respiratory Disease. Questionnaires were administered between July and October 2016, and the results were analyzed together with the baseline KOCOSS data. Based on the questionnaire data, patients were categorized into ACO and nonACO COPD groups according to the four sets of diagnostic criteria detailed above.

\section{Statistical analysis}

Statistical analyses were performed using SPSS software version 20.0 (IBM Corp., Armonk, NY, USA). Continuous data are expressed as the mean and range. Categorical variables were compared between the ACO and non-ACO COPD groups using the chi-squared test, and continuous variables were analyzed using Student's t test or the Mann-Whitney U test, depending on the normality of the data distribution. To evaluate changes in $\mathrm{FEV}_{1}$ and FVC over 3 years, mean delta values (difference between year 3 and baseline values) were compared between the ACO and non-ACO COPD groups using the independent $t$ test. The paired $t$ test was also used to analyze within-group changes in continuous variables. A $\mathrm{p}<0.05$ was considered significant in all analyses.

\section{Ethics statement}

Written informed consent was obtained from all patients enrolled in this study. Ethical approval was obtained from the ethics committees of each participating medical center.

\section{Results}

\section{Prevalence of ACO}

In total 1,475 patients evaluated: 202 of 1,475 (13.6\%), 32 of 1,475 (2.2\%), and 178 of 1,113 (16.0\%) were categorized as ACO according to the modified SEPAR, ATS Roundtable, and GINA/GOLD criteria, respectively. Furthermore, 305 of 1,250 patients (24.4\%) were categorized as ACO according to specialist diagnosis. The proportions of patients who did and did not satisfy the various diagnostic criteria for ACO are shown in Table 1. Regarding the modified SEPAR criteria, 87 patients met the major criteria and 125 patients met the minor criteria. Regarding the major criteria, $5.5 \%$ of the patients showed a 
Table 1. Diagnostic criteria of ACO among the study patients

\begin{tabular}{|c|c|c|}
\hline & Meet the criteria & Do not meet the criteria \\
\hline ACO according to ATS Roundtable & $32(2.2)$ & $1,443(97.8)$ \\
\hline \multicolumn{3}{|l|}{ Major } \\
\hline Smoking $\geq 10$ packs-year or exposure to air pollution $\geq 10 \mathrm{yr}$ & $1,183(80.2)$ & $292(19.8)$ \\
\hline History of asthma before $40 \mathrm{yr}$ & $76(6.4 \%)$ & $1,109(93.6)$ \\
\hline \multicolumn{3}{|l|}{ Minor } \\
\hline History of atopy or allergic rhinitis & $95(8.1)$ & $1,071(91.9)$ \\
\hline Separate BDR $\geq 12 \%$ and $200 \mathrm{~mL}$ & $47(11.8)$ & $350(88.2)$ \\
\hline Blood eosinophil count $\geq 300$ cells $/ \mu \mathrm{L}$ & $248(21.7)$ & $894(78.3)$ \\
\hline $\mathrm{BDR}>15 \%$ and $400 \mathrm{~mL}$ & $22(5.5)$ & $379(94.5)$ \\
\hline \multicolumn{3}{|l|}{ Minor criteria } \\
\hline IgE $>100$ IU, or history of atopy & $499(58.2)$ & $359(41.8)$ \\
\hline $\mathrm{BDR}>12 \%$ and $200 \mathrm{~mL}$ & $47(11.8)$ & $350(88.2)$ \\
\hline Blood eosinophil >5\% & $194(17.0)$ & $945(83.0)$ \\
\hline ACO according to GINA/GOLD checkbox & $178(16.0)$ & $935(84.0)$ \\
\hline Component of asthmatic feature $\geq 3$ & $191(17.2)$ & $922(82.8)$ \\
\hline Component of COPD feature $\geq 3$ & $1,068(96.0)$ & $45(4.0)$ \\
\hline ACO ( $\geq 3$ asthma and $\geq 3$ COPD features) & $178(16.0)$ & $935(84.0)$ \\
\hline
\end{tabular}

Values are presented as number (\%).

ACO: asthma-COPD overlap; ATS: American Thoracic Society; BD: bronchodilator; $\mathrm{FEV}_{1}$ : forced expiratory volume in one second; FVC: forced vital capacity; BDR: bronchodilator response; SEPAR: Spanish Society of Pneumonology and Thoracic Surgery; GINA: Global Initiative for Asthma; GOLD: Global Initiative for Chronic Obstructive Lung Disease; COPD: chronic obstructive pulmonary disease.

bronchodilator response $(\mathrm{BDR})>15 \% / 400 \mathrm{~mL}$. Regarding the minor criteria, $58.2 \%$ of the patients showed an IgE level $>100$ IU or a history of atopy, and $11.8 \%$ showed a BDR $>12 \% / 200$ $\mathrm{mL}$ on at least two separate occasions. In terms of blood eosinophils, $17.0 \%$ of the patients showed a baseline eosinophil count $>5 \%$. Regarding the ATS Roundtable criteria, 66 patients met the major criteria and 347 met the minor criteria. Regarding the major criteria, $80.2 \%$ of the patients had a smoking history of $\geq 10$ pack-years, and $5.6 \%$ showed a BDR $>400 \mathrm{~mL}$ $\left(\mathrm{FEV}_{1}\right)$. Regarding the minor criteria, $8.1 \%$ of the patients had a history of atopy or allergic rhinitis, $11.8 \%$ had two separate BDRs of $\geq 12 \% / 200 \mathrm{~mL}$, and $21.7 \%$ showed eosinophil counts $\geq 300$ cells $/ \mu \mathrm{L}$. Regarding the GINA/GOLD criteria, among 1,099 patients who responded to the asthma questionnaire, $17.2 \%$ responded positively to three or more asthma questions and $96.2 \%$ responded positively to three or more COPD questions. The number of patients who responded positively to a minimum of three questions for both asthma and COPD was $176(16.0 \%)$.

\section{Comparison of the clinical characteristics of the ACO} and non-ACO COPD groups according to the four sets of diagnostic criteria

Baseline clinical parameters including sex, age, body mass index (BMI), smoking history, and lung function tests were compared between the ACO and non-ACO COPD groups, defined according to the four sets of diagnostic criteria (Table 2). The ACO group defined according to the ATS Roundtable criteria showed no significant difference in the proportion of males, a significantly higher number of smoking pack-years, and a higher proportion of patients with a past history of asthma and atopy (52.5 vs. $41.7, \mathrm{p}=0.014 ; 65.6 \%$ vs. $4.8 \%$, $\mathrm{p}<0.001$; and $38.7 \%$ vs. $7.3 \%, \mathrm{p}<0.001$, respectively). The ACO group 


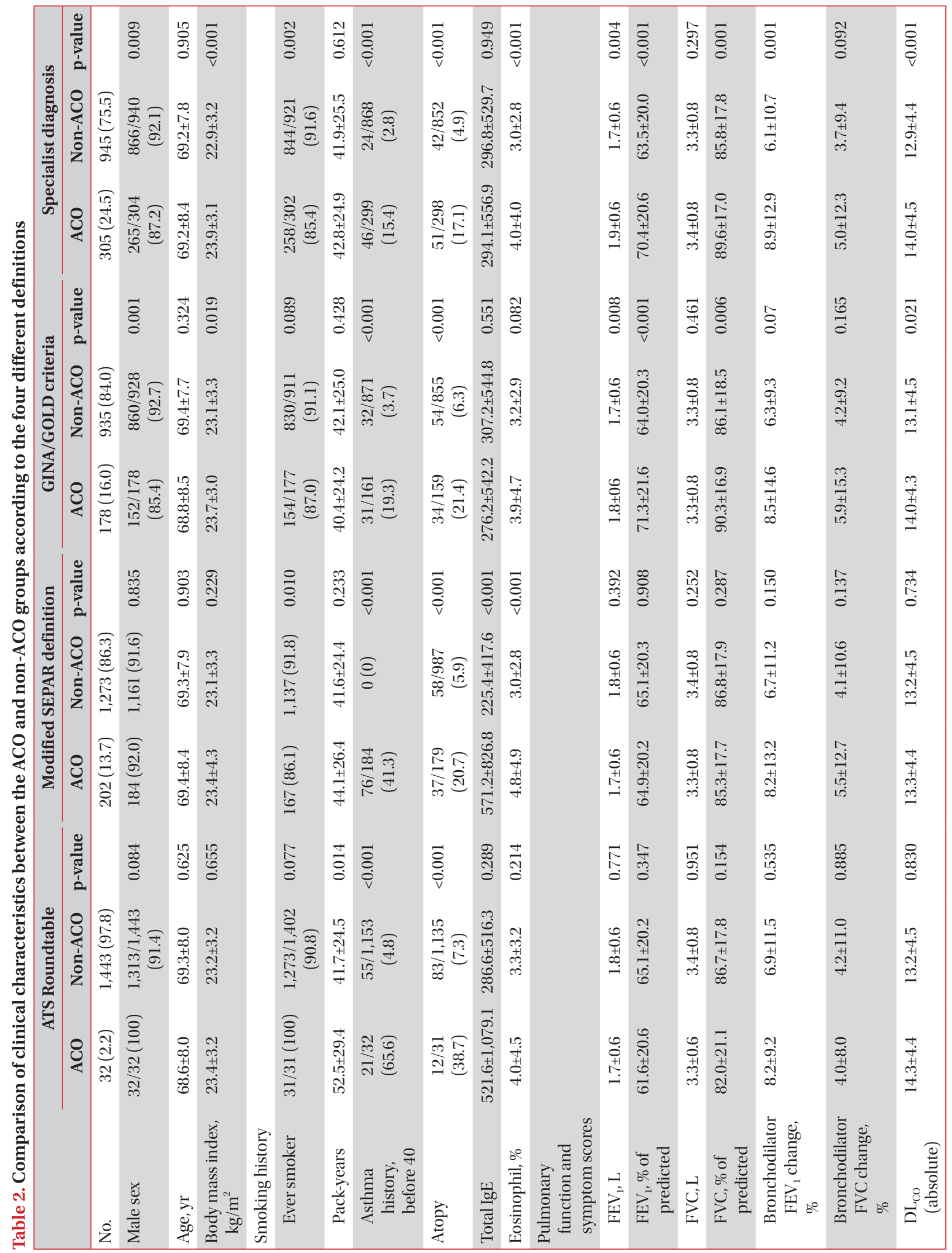




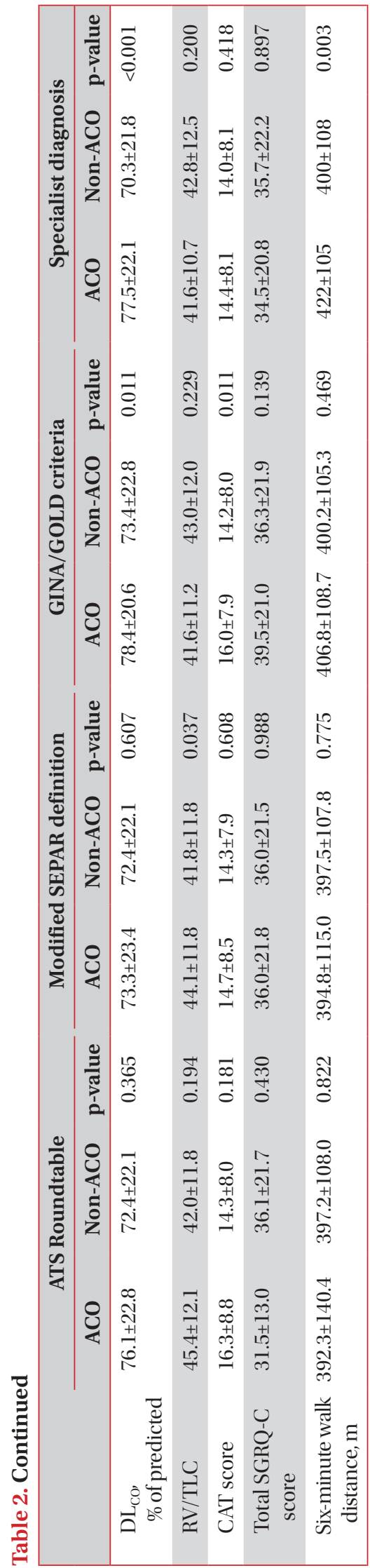

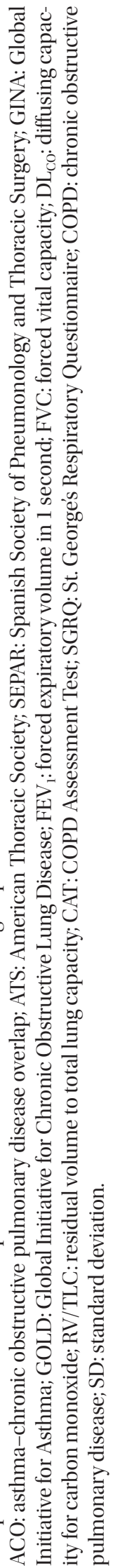

defined according to the modified SEPAR criteria showed no difference in the proportion of males, while showing a significantly lower proportion of patients with a smoking history, higher proportions of patients with a history of asthma and atopy, and a higher total IgE level and mean eosinophil count (\%) $(86.1 \%$ vs. $91.8 \%, \mathrm{p}=0.010 ; 41.3 \%$ vs. $0 \%, \mathrm{p}<0.001 ; 20.7 \%$ vs. $5.9 \%$, $\mathrm{p}<0.001 ; 571.2$ vs. 225.4 , $\mathrm{p}<0.001$; and $4.8 \%$ vs. $3.0 \%$, $\mathrm{p}<0.001$, respectively). The ACO group defined according to the GINA/GOLD criteria showed a significantly lower proportion of males, a higher BMI, and higher proportions of patients with a past history of asthma and atopy $(85.4 \%$ vs. $92.7 \%$, $\mathrm{p}=0.001 ; 23.7$ vs. $23.1, \mathrm{p}=0.019 ; 19.3 \%$ vs. $3.7 \%, \mathrm{p}<0.001$; and $21.4 \%$ vs. $6.3 \%$, $\mathrm{p}<0.001$, respectively). Relative to the non-ACO COPD group, the ACO group diagnosed according to specialists showed a significantly lower proportion of males, higher BMI, lower proportion of ever-smokers, higher proportion of patients with a past history of asthma and atopy, and a higher mean blood eosinophil count (\%) $(87.2 \%$ vs. $92.1 \%$, p=0.009; 23.9 vs. $22.9, \mathrm{p}<0.001 ; 85.4 \%$ vs. $91.6 \%, \mathrm{p}=0.002 ; 15.4 \%$ vs. $2.8 \%$, $\mathrm{p}<0.001 ; 17.1 \%$ vs. $4.9 \%$, $\mathrm{p}<0.001$; and $4.0 \%$ vs. $3.0 \%$, $\mathrm{p}<0.001$, respectively).

Regarding pulmonary function tests, the ACO group defined according to the ATS Roundtable criteria showed no significant difference in lung function compared to the nonACO COPD group. The ACO group defined according to the modified SEPAR criteria showed no significant difference in $\mathrm{FEV}_{1}$ or FVC compared to the non-ACO COPD group, but had a higher residual volume to total lung capacity (RV/TLC) ratio ( 44.1 vs. $41.8, \mathrm{p}=0.037$ ). When the GINA/GOLD criteria were applied, relative to the non-ACO COPD group, the ACO group showed higher $\mathrm{FEV}_{1}$ (absolute), FEV $(\%), \mathrm{FVC}$ (\%), diffusing capacity of the lung for carbon monoxide $\left(\mathrm{DL}_{\mathrm{CO}}\right)$ (absolute), and $\mathrm{DL}_{\mathrm{CO}}(\%)$ values ( 1.8 vs. $1.7, \mathrm{p}=0.008,71.3 \%$ vs. $64.0 \%$, $\mathrm{p}<0.001 ; 90.3 \%$ vs. $86.1 \%, \mathrm{p}=0.006 ; 14.0$ vs. $13.1, \mathrm{p}=0.021$; and $78.4 \%$ vs. $73.4 \%, p=0.011$, respectively). In terms of the COPD Assessment Test (CAT) score, the ACO group showed a higher score compared to the non-ACO COPD group (16.0 vs. $14.2, \mathrm{p}=0.011$ ). Relative to the non-ACO COPD group, the ACO group diagnosed according to specialists' opinions showed higher $\mathrm{FEV}_{1}(\%)$ and FVC (\%) values ( $70.4 \%$ vs. $63.5 \%$, $\mathrm{p}<0.001$ and $89.6 \%$ vs. $85.8 \%, \mathrm{p}=0.001$, respectively), and a higher $\mathrm{BDR}$ of $\mathrm{FEV}_{1}(8.9 \%$ vs. $6.1 \%, \mathrm{p}=0.001)$. They also showed better $\mathrm{DL}_{\mathrm{CO}}$ in absolute and percentage terms, and 6-minute walk distance result ( 14.0 vs. $12.9, \mathrm{p}<0.001 ; 77.5 \%$ vs. $70.3 \%$, $\mathrm{p}<0.001$; and 422 $\mathrm{m}$ vs. $400 \mathrm{~m}, \mathrm{p}=0.003$, respectively).

\section{Longitudinal changes in symptom scores}

During the 3-year follow-up, the total St. George's Respiratory Questionnaire (SGRQ) and CAT scores were compared between the ACO and non-ACO COPD groups defined according to the four sets of diagnostic criteria (Table 3). The ACO group defined according to the GINA/GOLD criteria showed 


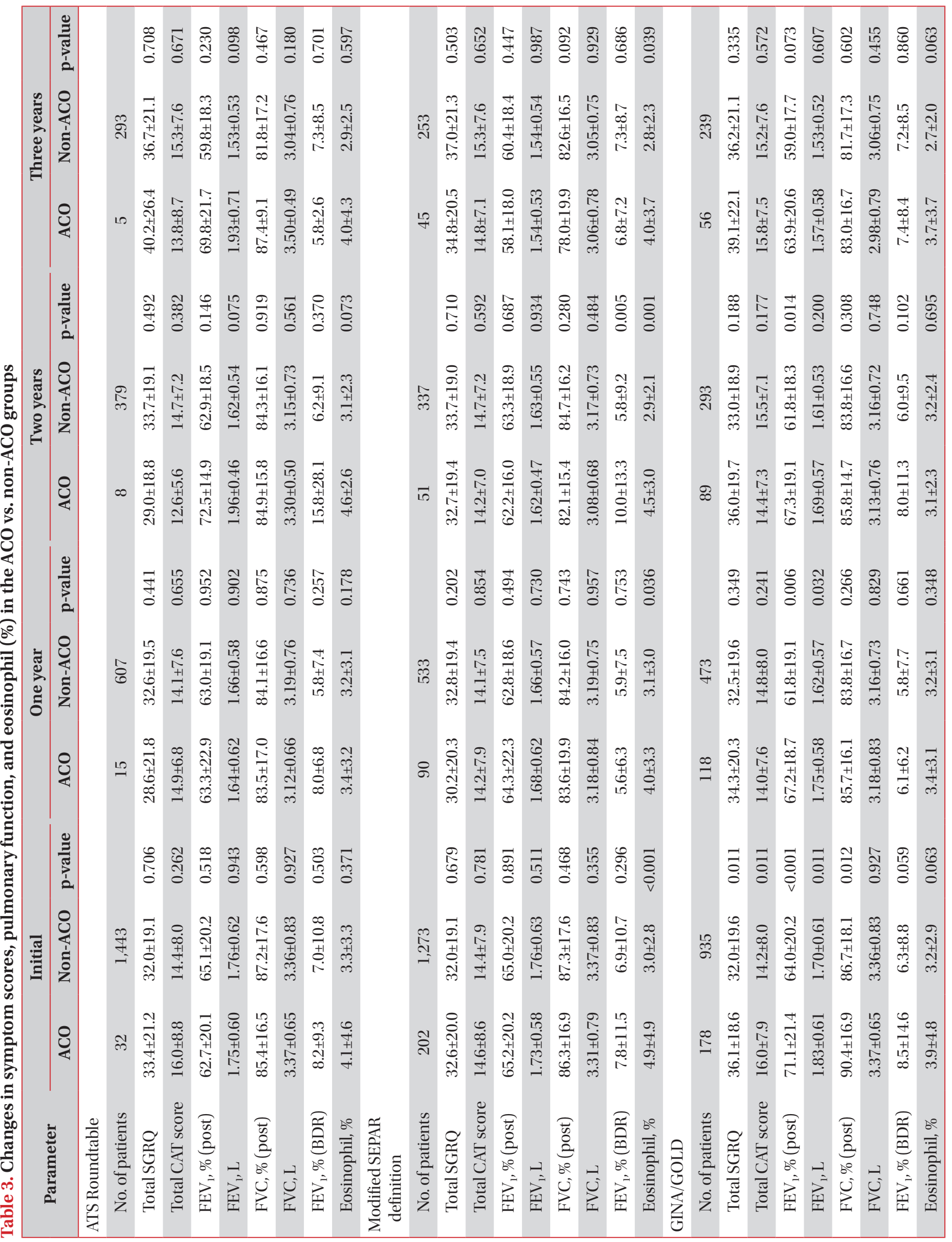




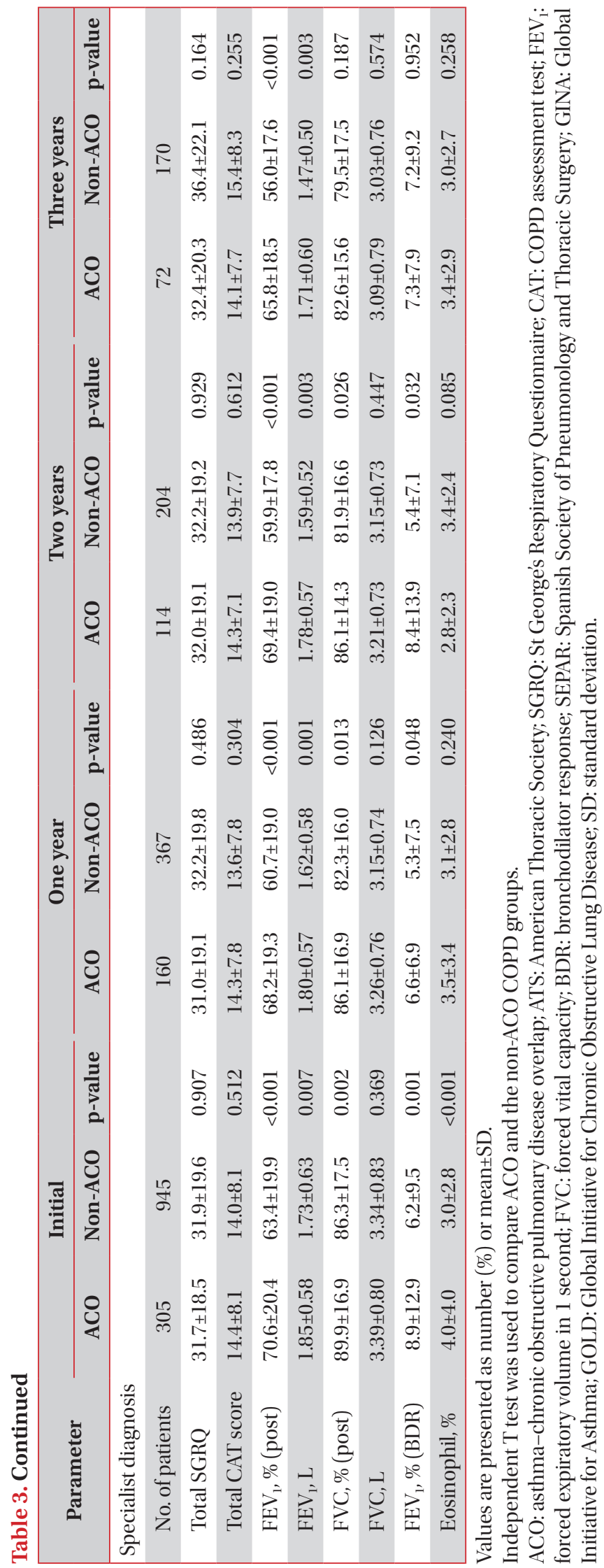


significantly higher SGRQ and CAT scores at baseline than the non-ACO COPD group (36.1 vs. $32.0, \mathrm{p}=0.011$ and 16.0 vs. 14.2, $\mathrm{p}=0.011$, respectively). However, during the 3 -year follow-up, no statistically significant difference was observed between the two groups. When the modified SEPAR, ATS Roundtable and specialists' diagnosis were applied, no statistically significant differences were found between the two groups, either at baseline or during follow-up.

\section{Longitudinal changes in pulmonary function and eosinophil count (\%)}

When the GINA/GOLD criteria were applied, $\mathrm{FEV}_{1}(\%)$ was significantly higher in the ACO group at baseline, and at the 1- and 2-year follow-ups, but not at the 3-year follow-up, compared to the non-ACO COPD group ( $71.1 \%$ vs. $64.0 \%$, $\mathrm{p}<0.001$; $67.2 \%$ vs. $61.8 \%$, $\mathrm{p}=0.006 ; 67.3 \%$ vs. $61.8 \%$, $\mathrm{p}=0.014$; and $63.9 \%$ vs. $59.0 \%, \mathrm{p}=0.073$, respectively). FVC (\%) was significantly higher in the ACO group only at baseline (90.4\% vs. $86.7 \%$; $\mathrm{p}=0.012$ ).

The ACO group defined according to the modified SEPAR criteria showed no significant difference in $\mathrm{FEV}_{1}(\%)$ or FVC (\%), either at baseline or during follow-up, compared to the non-ACO COPD group. The ACO group showed a higher BDR in terms of the $\mathrm{FEV}_{1}(\%)$ at the 2-year follow-up (10.0\% vs. 5.8\%, $\mathrm{p}=0.005)$. No significant group difference in $\mathrm{FEV}_{1}(\%)$, FVC (\%), or the BDR was seen when the ATS Roundtable criteria were applied. The ACO group defined based on specialists diagnosis showed a significantly higher $\mathrm{FEV}_{1}(\%)$ than the non-ACO COPD group, at baseline and at the 1-, 2-, and 3-year followups $(70.6 \%$ vs. $63.4 \%$, $\mathrm{p}<0.001 ; 68.2 \%$ vs. $60.7 \%$, $\mathrm{p}<0.001 ; 69.4 \%$ vs. $59.9 \%$, p $<0.001$; and $65.8 \%$ vs. $56.0 \%$, p $<0.001$, respectively). Regarding FVC (\%), the ACO group showed higher mean values, at baseline and at the 1- and 2-year follow-ups (89.9\%
A

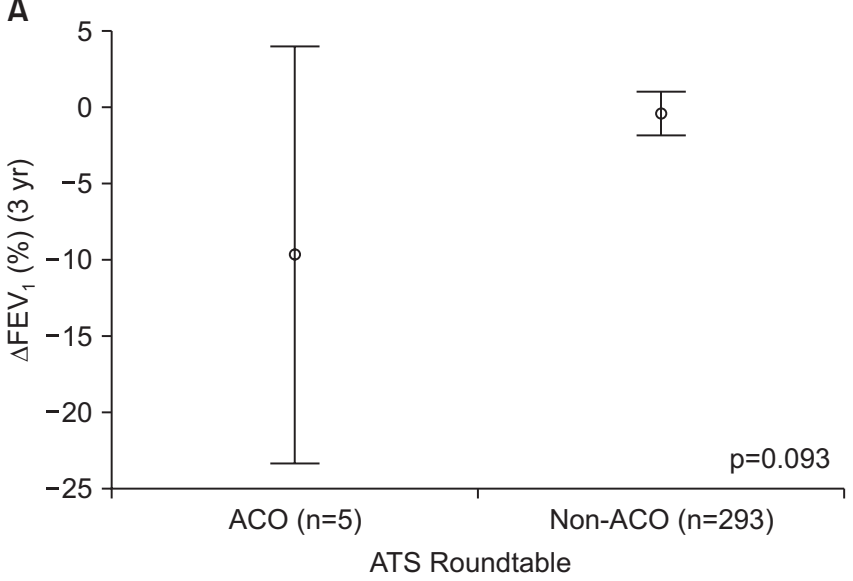

C

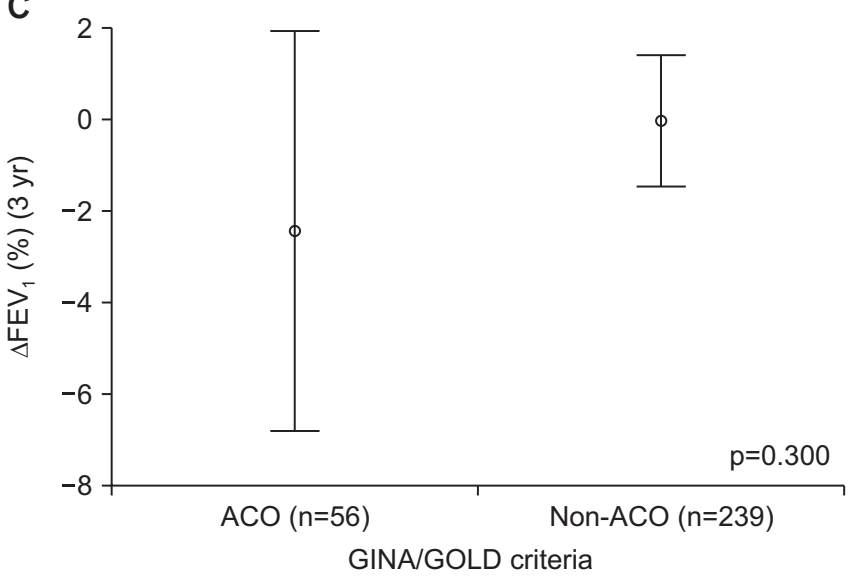

B

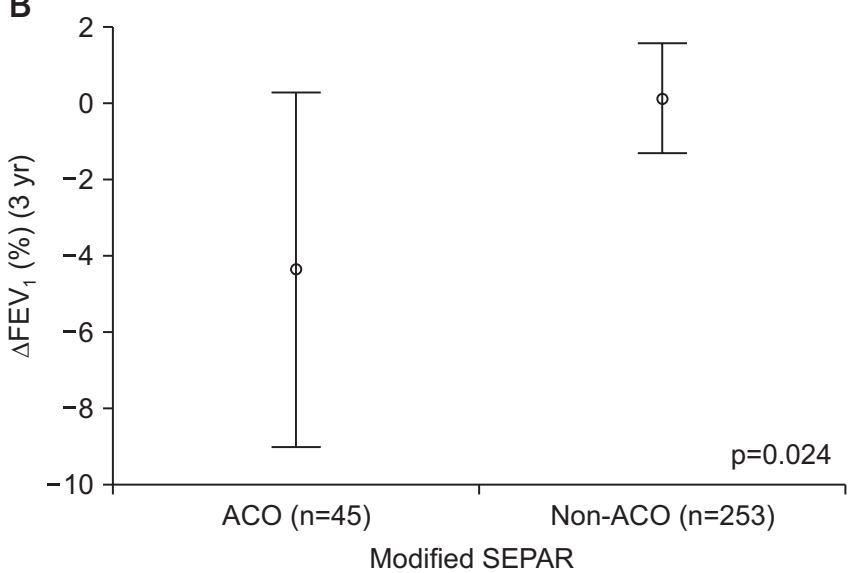

D

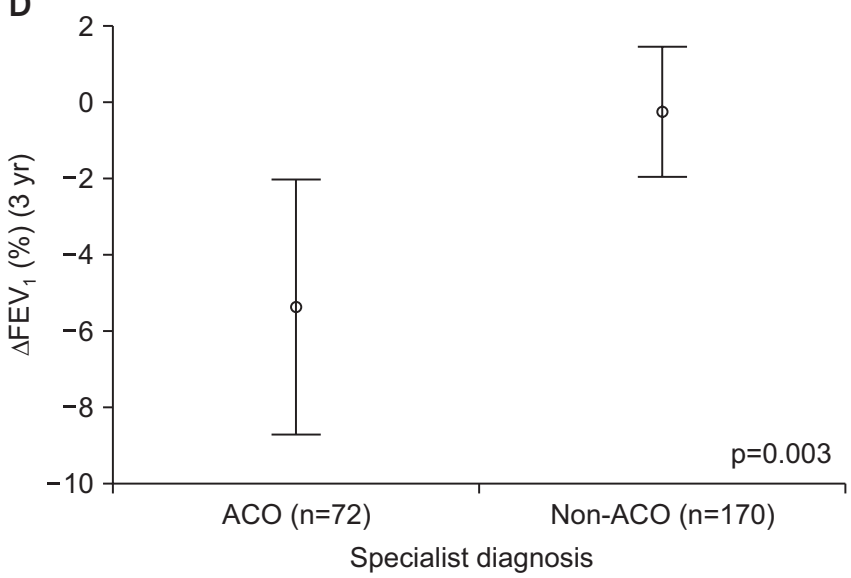

Figure 1. Comparison of mean values of three-year changes (with standard errors) in $\mathrm{FEV}_{1}(\%)$ between the ACO group and non-ACO COPD group according to ATS Roundtable (A), modified SEPAR (B), GINA/GOLD criteria (C), and specialist diagnosis (range, 2 standard error) (D).

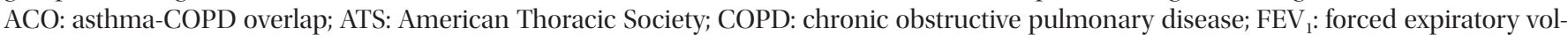
ume in 1 second; GINA: Global Initiative for Asthma; GOLD: Global Initiative for Chronic Obstructive Lung Disease; SEPAR: Spanish Society of Pneumonology and Thoracic Surgery. 
vs. $86.3 \%, \mathrm{p}=0.002 ; 86.1 \%$ vs. $82.3 \%, \mathrm{p}=0.013$; and $86.1 \%$ vs. $81.9 \%, \mathrm{p}=0.026$, respectively). The BDR in terms of the $\mathrm{FEV}_{1}$ (\%) was also higher in the ACO group, at baseline and at the 1 - and 2-year follow-ups ( $8.9 \%$ vs. $6.2 \%$, $\mathrm{p}=0.001 ; 6.6 \%$ vs. $5.3 \%$, $\mathrm{p}=0.048$; and $8.4 \%$ vs. $5.4 \%$, $\mathrm{p}=0.032$, respectively).

When the GINA/GOLD and ATS Roundtable criteria were applied, no significant difference in blood eosinophils (\%) was found between the ACO and non-ACO COPD group. The ACO group defined by the modified SEPAR criteria showed a significantly higher blood eosinophil count (\%), at baseline and at the 1-, 2-, and 3-year follow-ups ( $4.9 \%$ vs. 3.0\%, p $<0.001$; $4.0 \%$ vs. $3.1 \%$, $\mathrm{p}=0.036 ; 4.5 \%$ vs. $2.9 \%, \mathrm{p}=0.001$; and $4.0 \%$ vs. $2.8 \%$, $\mathrm{p}=0.039$, respectively). The ACO group defined based on specialist diagnosis showed a higher blood eosinophil count (\%) only at baseline ( $4.0 \%$ vs. $3.0 \%, \mathrm{p}<0.001)$.

A

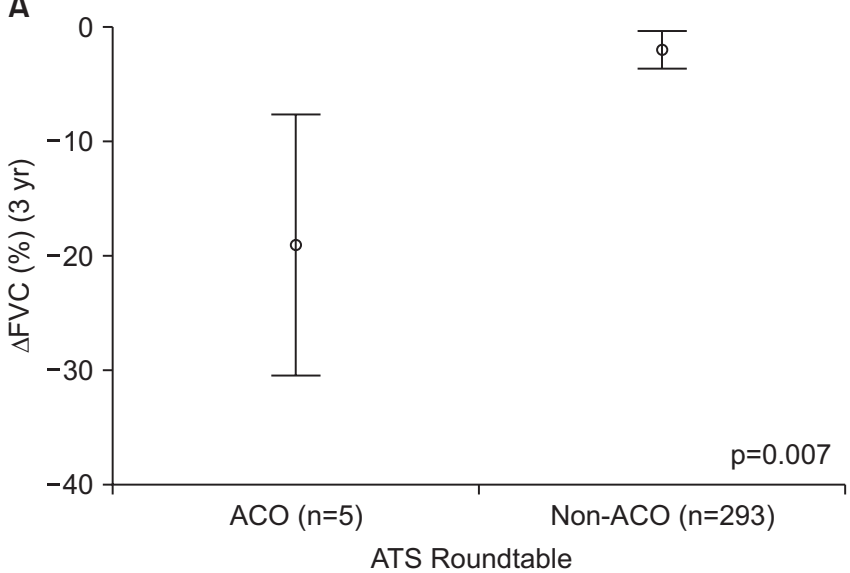

C

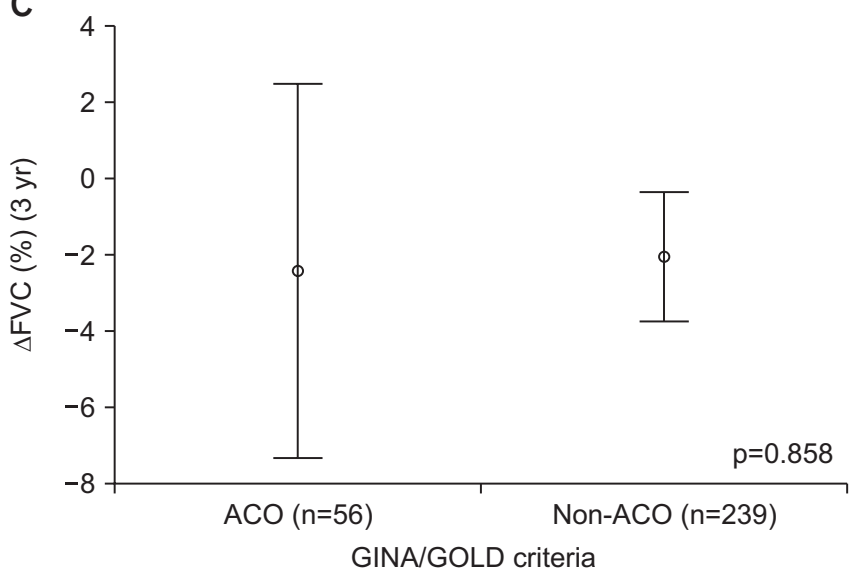

\section{Change in lung function over 3 years}

The changes in $\mathrm{FEV}_{1}$ and $\mathrm{FVC}$ over the 3-year follow-up were compared between the ACO and non-ACO COPD groups according to the four sets of ACO diagnostic criteria (Supplementary Table S2). The ACO group defined by the ATS Roundtable showed significantly larger decrease in percentage and absolute FVC values compared to the non-ACO COPD group $(-18.9 \%$ vs. $-2.2 \%$, $\mathrm{p}=0.007$; and $-412 \mathrm{~mL}$ vs. $-17 \mathrm{~mL}, \mathrm{p}=0.036$ ), while both the ACO and non-ACO COPD groups showed significant within-group decreases $(\mathrm{p}=0.03$ and $p=0.011$, respectively) (Figure 1 ). When the modified SEPAR definition was applied, the ACO group showed a significantly larger decrease in $\mathrm{FEV}_{1}(\%)(-4.3 \%$ vs. $0.2 \%, \mathrm{p}=0.024)$. When the GINA/GOLD criteria were applied, the two groups did not show a significant difference in the extent of change in pulmonary function parameters over the 3-year follow-up
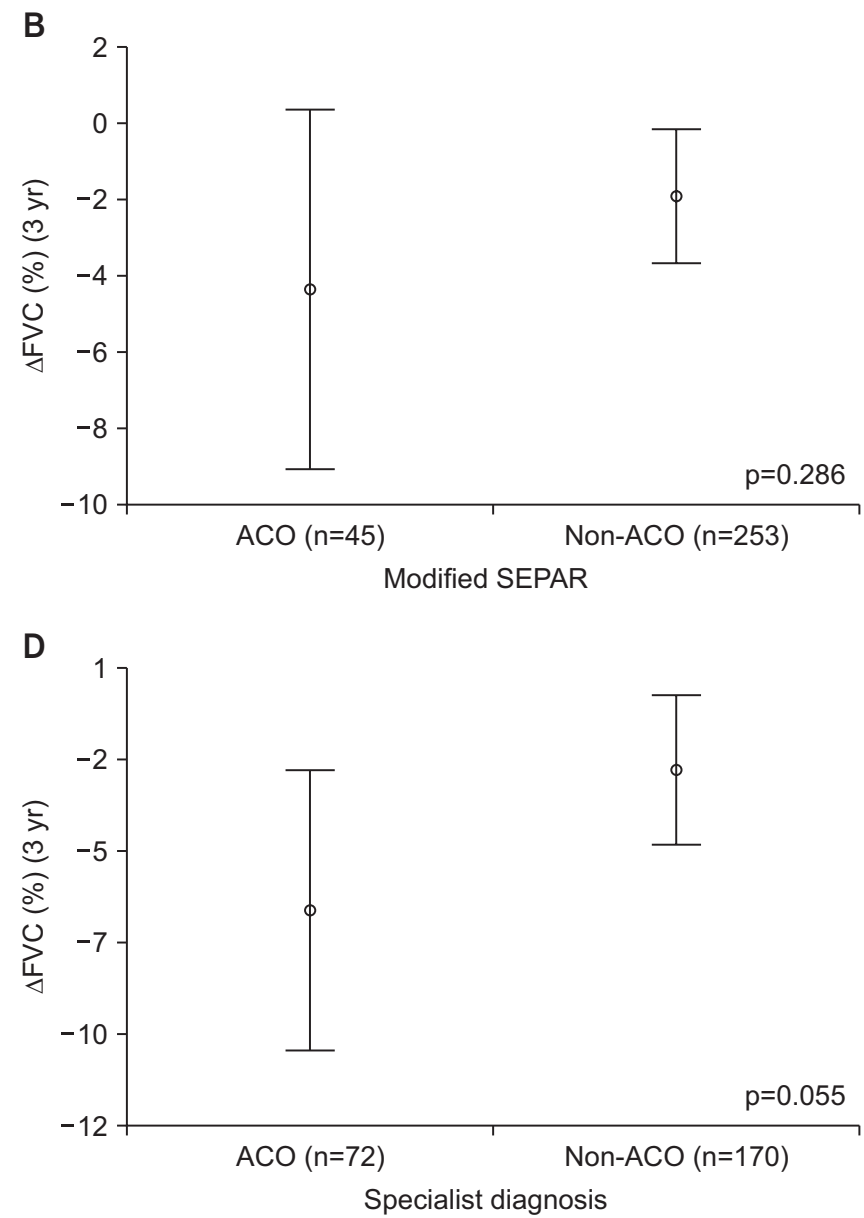

Figure 2. Comparison of mean values of three-year changes (with standard errors) in FVC (\%) between the ACO group and non-ACO COPD group according to ATS Roundtable (A), modified SEPAR (B), GINA/GOLD criteria (C), and specialist diagnosis (D) (range, 2 standard error). ACO: asthma-COPD overlap; ATS: American Thoracic Society; COPD: chronic obstructive pulmonary disease; FVC: forced vital capacity; GINA: Global Initiative for Asthma; GOLD: Global Initiative for Chronic Obstructive Lung Disease; SEPAR: Spanish Society of Pneumonology and Thoracic Surgery. 
(Figure 2). Finally, the ACO group diagnosed by specialists showed a significantly larger decrease in the $\mathrm{FEV}_{1}(\%)$ compared to the non-ACO group ( $-5.4 \%$ vs. $-0.2 \%$, $\mathrm{p}=0.003$ ) (Figures 1 and 2).

\section{Discussion}

This retrospective cohort study compared longitudinal changes in lung function and symptoms between ACO and non-ACO COPD groups defined according to four sets of diagnostic criteria. The results showed that the prevalence of ACO and outcomes varied according to the diagnostic criteria applied. In terms of clinical characteristics, ACO defined by specialists (based on clinical experience) and the GINA/GOLD criteria showed similar results, while ACO defined according to the ATS Roundtable criteria showed results that were inconsistent with those of the other diagnostic criteria.

Considering that the prevalence of ACO among COPD patients is $15 \%-30 \%^{12-14}$, the prevalence of $3.3 \%$ calculated herein based on the ATS Roundtable was relatively low. This may be due to the strictness of criteria for ACO diagnosis, i.e., fulfillment of all three major criteria for ACO plus at least one minor criterion. The BDR was included among both the major and minor criteria, such that patients with no BDR (but with other asthmatic features) and patients without BDR test results are less likely to be included in the ACO group. Based on the modified SEPAR definition, $13.5 \%$ of our patients were categorized as ACO; this is similar to the study by Cosio et al. ${ }^{13}$, in which the prevalence of ACO was $15 \%$. Inoue et al. ${ }^{15}$ reported that $16.6 \%$ of their patients diagnosed with COPD had syndromic features of ACO according to the GINA/GOLD criteria. Kobayashi et al. ${ }^{16}$, using the same diagnostic criteria, reported that $14.4 \%$ of patients with COPD had ACO. These results are similar to those of our study, in which $16.0 \%$ of the patients were defined as ACO according to the GINA/GOLD criteria.

In terms of baseline clinical characteristics, the ACO groups defined according to the different criteria had clinical features similar to those of the non-ACO COPD groups. The ACO groups included lower proportions of males and ever-smokers, and showed higher BMI values and eosinophil counts (\%) than the non-ACO COPD groups. The age differences between the ACO and non-ACO groups defined according to each category was not evident. This is contrary to the previous studies by Kobayashi et al. ${ }^{16}$, and Inoue et al. ${ }^{15}$, in which the ACO groups showed significantly younger mean age when compared to the non-ACO groups. In addition, the ACO groups had significantly higher proportions of patients with a past history of asthma and atopy, as reported in previous studies on $\mathrm{ACO}^{16,17}$. Compared to the other ACO criteria, the ACO group defined according to our criteria showed clinical characteristics more consistent with those reported in previous studies on ACO, including a significantly higher BDR and blood eosinophil count $(\%)^{8,18}$. The total IgE levels of the groups varied according to the diagnostic criteria applied; moreover, total IgE level was not included in all of the diagnostic criteria.

The ACO groups defined according to the modified SEPAR criteria and specialists diagnosis showed markedly larger decreases in $\mathrm{FEV}_{1}$ during the 3-year follow-up compared to the non-ACO COPD group. The ACO group defined according to the GINA/GOLD and ATS Roundtable criteria also showed a tendency toward a decline in $\mathrm{FEV}_{1}$ over the 3-year follow-up period. Lange et al. ${ }^{10}$ reported that COPD patients with asthmatic features showed a higher rate of annual decline in $\mathrm{FEV}_{1}$, and Tkacova et al. ${ }^{9}$ showed that ACO patients with bronchial hyperresponsiveness exhibited a faster decline in $\mathrm{FEV}_{1}$.

In all criteria, FVC decrease was more evident in the ACO groups than in the non-ACO COPD groups. We assume this change may be related to underlying diseases including cardiovascular diseases. Decreased FVC is associated with increased cardiovascular risk, especially heart failure, and underlying cardiovascular disease can also affect prognosis of $\mathrm{COPD}^{19,20}$. In the study by Ingebrigtsen et al. ${ }^{21}$, the heart failure admission was more frequent in the ACO group when compare to the non-ACO COPD group. Nevertheless, underlying diseases were not evaluated in this study. This correlation is an assumption and requires a further validation in a future study.

Regarding RV/TLC, which is a parameter reported to be associated with air trapping in $\mathrm{COPD}^{22}$, the ACO group defined according to modified SEPAR criteria showed significantly higher RV/TLC than the non-ACO COPD group in the present study. We assume that this finding may be due to the inclusion of BDR in modified SEPAR criteria. In the study by Gao et al. ${ }^{23}$, the ACO group showed significant difference in air trapping between prebronchodialtor and postbronchodilator values, suggesting the possibility of high variability. Further studies are required for a more accurate evaluation of air trapping indexes in ACO.

The change of FVC in 3 follow-up years was large in the ACO group defined by ATS Roundtable criteria, with a mean decrease of $412 \mathrm{~mL}$ in 3 years. It should be taken into account that the number of patients defined as the ACO group with three- year data were too small to produce a reliable result. For a more accurate analysis, much larger number of patients are necessary. Another assumption is that air trapping has increased over 3 years in the ACO group defined by ATS Roundtable criteria. Suggested by a larger baseline value of RV/TLC in the ACO group, air trapping may have increased over 3 years and influenced FVC. However, the serial analysis of RV/TLC was not performed in our study, and a future study evaluating the interrelationship between longitudinal changes of both RV/TLC and FVC is necessary.

This study had several limitations. First, data on previous 
history of asthma were acquired via a questionnaire that provided relatively little information on the patient's past medical history, which may have affected the ACO prevalence rate. Second, changes in pulmonary function were assessed only in terms of the difference between the baseline and 3-year follow-up, so the rate of decline could not be calculated. Finally, no analysis of mortality was performed.

Based on the results of this study, we believe that the ATS Roundtable diagnostic criteria for ACO may be inappropriate for clinical use in Korea due to their strictness. The GINA/ GOLD and modified SEPAR criteria were similarly effective for diagnosing ACO, although the modified SEPAR criteria were superior in terms of capturing longitudinal changes in SGRQ and CAT scores. Furthermore, despite the variability among the four sets of diagnostic criteria, the ACO group always showed an overall tendency towards a more rapid decline in lung function compared to the non-ACO COPD group, such that more intensive airway management is necessary in these patients.

In the present study, prevalence and clinical characteristics of ACO varied depending on the diagnostic criteria applied. The disparities in clinical presentation and longitudinal outcomes may have resulted from different distributions of several distinct phenotypes within ACO groups, and in this context, defining ACO by specialist diagnosis may be more comprehensive and practical in clinical application.

\section{*Author Affiliations}

${ }^{1}$ Division of Pulmonary and Critical Care Medicine, Department of Internal Medicine, Yeouido St. Mary's Hospital, College of Medicine, The Catholic University of Korea, Seoul, ${ }^{2} \mathrm{Di}-$ vision of Pulmonary and Critical Care Medicine, Department of Internal Medicine, Seoul Metropolitan Government Seoul National University Boramae Medical Center, Seoul, ${ }^{3}$ Division of Pulmonary, Allergy and Critical Care Medicine, Department of Internal Medicine, Hallym University Chuncheon Sacred Heart Hospital, Hallym University College of Medicine, Chuncheon, ${ }^{4}$ Division of Pulmonary, Allergy and Critical Care Medicine, Department of Internal Medicine, Hallym University Sacred Heart Hospital, Hallym University College of Medicine, Anyang, ${ }^{5}$ Regional Center for Respiratory Disease, Yeungnam University Medical Center, Yeungnam University College of Medicine, Daegu, ${ }^{6}$ Division of Respiratory and Critical Care Medicine, Department of Internal Medicine, Korea University College of Medicine, Seoul, ${ }^{7}$ Department of Internal Medicine, Korea University Anam Hospital, Korea University College of Medicine, Seoul, ${ }^{8}$ Division of Pulmonary, Allergy and Critical Care Medicine, Department of Internal Medicine, Seoul St. Mary's Hospital, College of Medicine, The Catholic University of Korea, Seoul, ${ }^{9}$ Division of Pulmonary, Allergy and Critical Care Medicine, Department of Internal Medicine,
Konkuk University School of Medicine, Seoul, Republic of Korea

\section{Authors' Contributions}

Conceptualization: Lim JU, Yoon HK. Methodology: Lim JU, Yoon HK. Formal analysis: Lim JU. Data curation: Kim DK, Lee MG, Hwang YI, Shin KC, In KH, Lee SY, Rhee CK, Yoo KH. Software: Lim JU. Validation: Yoon HK. Investigation: Lim JU, Yoon HK. Writing - original draft preparation: Lim JU. Writing - review and editing: Rhee CK, Yoon HK. Approval of final manuscript: all authors.

\section{Conflicts of Interest}

No potential conflict of interest relevant to this article was reported.

\section{Funding}

This research was supported by grants from the Research of Korea Centers for Disease Control and Prevention (2016ER670102 and 2018ER670100), and by a grant from the Korean Academy of Tuberculosis and Respiratory Diseases (KATRD-S-2017-1).

\section{Supplementary Material}

Supplementary material can be found in the journal homepage (http://www.e-trd.org).

Supplementary Table S1. Criteria for diagnosis of ACO.

Supplementary Table S2. Change in lung function in 3 years.

\section{References}

1. Yamamura K, Hara J, Kobayashi T, Ohkura N, Abo M, Akasaki $\mathrm{K}$, et al. The prevalence and clinical features of asthma-COPD overlap (ACO) definitively diagnosed according to the Japanese Respiratory Society Guidelines for the Management of ACO 2018. J Med Invest 2019;66:157-64.

2. Gibson PG, Simpson JL. The overlap syndrome of asthma and COPD: what are its features and how important is it? Thorax 2009;64:728-35.

3. Sin DD, Miravitlles M, Mannino DM, Soriano JB, Price D, Celli $\mathrm{BR}$, et al. What is asthma-COPD overlap syndrome? Towards a consensus definition from a round table discussion. Eur Respir J 2016;48:664-73.

4. Soler-Cataluna JJ, Cosio B, Izquierdo JL, Lopez-Campos JL, 
Marin JM, Aguero R, et al. Consensus document on the overlap phenotype COPD-asthma in COPD. Arch Bronconeumol 2012;48:331-7.

5. Sandfeld-Paulsen B, Meldgaard P, Sorensen BS, Safwat A, Aggerholm-Pedersen N. The prognostic role of inflammationscores on overall survival in lung cancer patients. Acta Oncol 2019;58:371-6.

6. 2018 Exceptional surveillance of asthma: diagnosis, monitoring and chronic asthma management (NICE guideline NG80). London: National Institute for Health and Care Excellence; 2018.

7. Park HY, Lee SY, Kang D, Cho J, Lee H, Lim SY, et al. Favorable longitudinal change of lung function in patients with asthmaCOPD overlap from a COPD cohort. Respir Res 2018;19:36.

8. Joo H, Han D, Lee JH, Rhee CK. Heterogeneity of asthmaCOPD overlap syndrome. Int J Chron Obstruct Pulmon Dis 2017;12:697-703.

9. Tkacova R, Dai DL, Vonk JM, Leung JM, Hiemstra PS, van den Berge M, et al. Airway hyperresponsiveness in chronic obstructive pulmonary disease: a marker of asthma-chronic obstructive pulmonary disease overlap syndrome? J Allergy Clin Immunol 2016;138:1571-9.

10. Lange P, Colak Y, Ingebrigtsen TS, Vestbo J, Marott JL. Longterm prognosis of asthma, chronic obstructive pulmonary disease, and asthma-chronic obstructive pulmonary disease overlap in the Copenhagen City Heart study: a prospective population-based analysis. Lancet Respir Med 2016;4:454-62.

11. Lee JY, Chon GR, Rhee CK, Kim DK, Yoon HK, Lee JH, et al. Characteristics of patients with chronic obstructive pulmonary disease at the first visit to a pulmonary medical center in Korea: The KOrea COpd Subgroup Study Team Cohort. J Korean Med Sci 2016;31:553-60.

12. Alshabanat A, Zafari Z, Albanyan O, Dairi M, FitzGerald JM. Asthma and COPD overlap syndrome (ACOS): a systematic review and meta analysis. PLoS One 2015;10:e0136065.

13. Cosio BG, Soriano JB, Lopez-Campos JL, Calle-Rubio M, Soler-Cataluna JJ, de-Torres JP, et al. Defining the asthma-COPD overlap syndrome in a COPD cohort. Chest 2016;149:45-52.

14. Tho NV, Park HY, Nakano Y. Asthma-COPD overlap syndrome (ACOS): a diagnostic challenge. Respirology 2016;21:410-8.

15. Inoue $H$, Nagase $T$, Morita S, Yoshida A, Jinnai T, Ichinose $\mathrm{M}$. Prevalence and characteristics of asthma-COPD overlap syndrome identified by a stepwise approach. Int J Chron Obstruct Pulmon Dis 2017;12:1803-10.

16. Kobayashi S, Hanagama M, Yamanda S, Ishida M, Yanai M. Inflammatory biomarkers in asthma-COPD overlap syndrome. Int J Chron Obstruct Pulmon Dis 2016;11:2117-23.

17. Kauppi P, Kupiainen H, Lindqvist A, Tammilehto L, Kilpelainen M, Kinnula VL, et al. Overlap syndrome of asthma and COPD predicts low quality of life. J Asthma 2011;48:279-85.

18. de Marco R, Pesce G, Marcon A, Accordini S, Antonicelli L, Bugiani $\mathrm{M}$, et al. The coexistence of asthma and chronic obstructive pulmonary disease (COPD): prevalence and risk factors in young, middle-aged and elderly people from the general population. PLoS One 2013;8:e62985.

19. Rabe KF, Hurst JR, Suissa S. Cardiovascular disease and COPD: dangerous liaisons? Eur Respir Rev 2018;27:180057.

20. Silvestre OM, Nadruz W Jr, Querejeta Roca G, Claggett B, Solomon SD, Mirabelli MC, et al. Declining lung function and cardiovascular risk: the ARIC study. J Am Coll Cardiol 2018; 72:1109-22.

21. Ingebrigtsen TS, Marott JL, Vestbo J, Nordestgaard BG, Lange P. Coronary heart disease and heart failure in asthma, COPD and asthma-COPD overlap. BMJ Open Respir Res 2020;7:e000470.

22. Vestbo J, Hurd SS, Agusti AG, Jones PW, Vogelmeier C, Anzueto A, et al. Global strategy for the diagnosis, management, and prevention of chronic obstructive pulmonary disease: GOLD executive summary. Am J Respir Crit Care Med 2013;187: 347-65.

23. Gao Y, Zhai X, Li K, Zhang H, Wang Y, Lu Y, et al. Asthma COPD overlap syndrome on CT densitometry: a distinct phenotype from COPD. COPD 2016;13:471-6. 